\title{
L’agriculture familiale : rengaine ou nouveauté ?
}

\author{
Philippe Lacombe \\ Agroéconomiste, INRA, 75007 Paris, France
}

Les Nations unies ont fait de $2014 l^{\prime}$ « Année internationale de l'agriculture familiale ». La communauté internationale du domaine, tout particulièrement la recherche agronomique pour le développement, a alors multiplié manifestations et ouvrages. L'agroéconomiste Philippe Lacombe, l'un des spécialistes de ces sujets, propose ici une analyse critique des réflexions multiformes sur l'agriculture familiale qui ont été avancées et débattues à cette occasion, en particulier sur l'actualité et le futur de cette question ainsi que sur les modes d'approche d'une réalité incontournable, l'agriculture familiale représentant plus de huit exploitations sur dix dans le monde.

La Rédaction

\section{Mots-clés :}

agriculture familiale ; politique publique ; territoire ; produit ; organisations paysannes ; ménages agricoles; structures agricoles

\section{Keywords:}

family farming; political economy; places and people; products; family farm organizations; farm households; farming structures

\begin{abstract}
Résumé - L'année 2014, retenue par les organisations internationales comme année de l'agriculture familiale, a été l'occasion pour plusieurs équipes françaises de recherche engagées sur ce thème de livrer une synthèse de leurs travaux et de réfléchir sur leur contribution à la compréhension, l'évaluation, la gouvernance de cette forme d'organisation agraire considérée, tantôt comme archaïque, tantôt comme performante. Le propos de cet article est de situer ces travaux dans le mouvement des idées sur l'agriculture familiale. Ces recherches, en analysant l'importance, la diversité et les potentialités de l'agriculture familiale, en viennent à mettre opportunément l'accent sur les conditions et modalités de son insertion efficace dans les sociétés contemporaines. L'action publique et les décisions privées s'en trouvent éclairées. Plus généralement, ces travaux renouvellent l'analyse du système productif agricole.
\end{abstract}

\begin{abstract}
Family farming: catchphrase or novelty? The year 2014, declared by international organizations as the Year of Family Farming, offered an opportunity for several French research teams engaged on this theme to produce a summary of their work and to reflect on their contributions to the understanding, evaluation and governance of this form of agrarian organization now regarded at times as archaic, at times as successful. The aim of this article is to position these works within the current trends of ideas on family farming. By analyzing the size, diversity and potential of family farming, these studies opportunely focus on the terms and conditions of its effective integration in contemporary societies. As a result, they contribute to inform public policies and private decisions. More generally, these studies offer new perspectives for the analysis of the agricultural production system.
\end{abstract}

L'ONU a déclaré 2014 « Année internationale de l'agriculture familiale ». Cette initiative a donné lieu à de multiples manifestations qui ont mobilisé divers milieux sociaux : professions, administrations, collectivités publiques, ONG, associations, écoles..., lesquels ont communiqué leurs témoignages, expériences, soutiens, propositions, interrogations à propos de l'agriculture familiale. La recherche scientifique, notamment dans le champ des sciences sociales, a apporté son concours à ces échanges. Il est vrai que ce thème est, pour ces sciences, une vieille question (Petit, 1975, 2006) : en se limitant à la période contemporaine, la thèse d'Augé-Laribé lui était déjà consacrée (Augé-Laribé, 1902), les manuels d'économie rurale ne manquent pas d'y faire référence (Milhau et Montagne, 1964 ; Badouin, 1971), y voyant même parfois une caractéristique fondamentale et essentielle de l'activité agricole. Les sociologues, après avoir analysé la «fin des paysans » (Mendras, 1967, 1984), étudient leurs successeurs (Hervieu et Purseigle, 2013), voire leur retour (Van der Ploeg, 2014). Les historiens, eux aussi, signalent la permanence du thème (Barral, 1968 ; Mayaud, 1999). Les essais sur les transformations

Auteur correspondant : philber.lacombe@aliceadsl.fr 
agricoles contemporaines, françaises (Chombart de Lauwe, 1979 ; Servolin, 1989 ; Malassis, 2001) ou mondiales (Neveu, 2012 ; Loyat, 2013 ; Mazoyer et Roudart, 2005 ; Petit, 2011), soulignent les rôles des exploitations agricoles familiales (EAF), leurs atouts, leurs handicaps.

L'Église catholique valorise régulièrement ce type d'exploitation (Abbaye Saint-Pierre de Solesmes, 1960). Les partis politiques ont souvent manifesté un intérêt pour elle, ce qui ne manque pas de laisser entrevoir ses ambiguïtés. La droite affiche généralement son soutien à la famille et à la propriété, la gauche, après s'être divisée sur l'alternative exploitation privée ou collective, reconnaît, elle aussi, l'exploitation agricole familiale comme une forme non capitaliste durable, rejoignant ainsi la position défendue par Jean Jaurès dès le début du $X X^{\mathrm{e}}$ siècle (Pech, 2009). Les formes d'agriculture collective (kolkhoze, sovkhoze, domaine autogéré, coopérative de production, ferme d'État, combinat, kibboutz...), après avoir pourtant retenu l'attention au cours du $\mathrm{XX}$ e siècle, ont disparu des débats ; c'est leur remplacement qui, aujourd'hui, mobilise les énergies. Le thème de l'agriculture familiale ne manque donc pas d'antécédents notables et l'actualité immédiate voit revenir et s'intensifier le débat sur sa place et son rôle.

D’un côté, le mouvement général de libéralisation économique, l'ajustement structurel, la valorisation d'une agriculture "market oriented », la financiarisation de l'agriculture soulignent la puissance des forces adverses à l'agriculture familiale : on parle de guerre (Pouch, 2010), on annonce un désastre (Neveu, 2012)... Les cessions d'actifs agricoles inquiètent et leur encadrement est suggéré (CAS, 2010). L'organisation des familles, en se diversifiant et se recomposant (Segalen, 2012 ; Rémy, 2010), met en question l'agriculture familiale, les liens exploitation/famille se distendent au point que la législation encourage la distinction, voire la séparation, entre les deux. La crise alimentaire mondiale de la fin des années 2000, les émeutes de la faim, l'évolution chaotique des prix, les pratiques spéculatives n'ont pas entamé la confiance dans les mécanismes du marché, bien qu'on ait pu parler d'un retour de la question agricole (Hervieu et al., 2010 ; Cornu et Mayaud, 2008).

D'un autre côté, un récent rapport de la Banque mondiale (2008), le rapport de l'International Assessment of Agricultural Science and Technology for Development (McIntyre et al., 2009) et la décision de l'ONU dédiant l'année 2014 à l'agriculture familiale, invitent à s'intéresser, au nom de préoccupations actuelles (sécurité alimentaire, multifonctionnalité, pauvreté, emploi, environnement...), à l'agriculture dans l'état où elle se trouve dans le monde, c'est-à-dire largement familiale. Parfois, la politique agricole affirme explicitement une référence positive en faveur de l'exploitation familiale ; en France, les ministres successifs de l'Agriculture ont ainsi régulièrement déclaré cette préférence (Roux, 2014).
On en vient alors à se demander si cette forme d'agriculture est un archaïsme obsolète, un retour, une rengaine ou une nouveauté, une opportunité ou une diversion. La réponse à cette question nous paraît utilement éclairée par une série de publications qui, dans ce paysage, très rapidement rappelé ici, sont particulièrement bienvenues. Dans l'impossibilité matérielle d'analyser chaque ouvrage pris individuellement, on trouvera leurs références dans l'encadré page suivante. On se propose de les situer collectivement dans l'histoire des idées concernant l'agriculture familiale, en raison de la proximité et de la complémentarité entre ces publications, dans lesquelles on retrouve souvent les mêmes auteurs. On souhaite ainsi dégager, dans un premier temps, les constats qui nous paraissent marquer une étape dans l'évolution des idées; les premiers s'intéressent à l'exploitation agricole familiale et complètent les acquis déjà disponibles sur son organisation et son fonctionnement ; les seconds, plus novateurs à nos yeux, concernent l'insertion de cette forme d'exploitation dans son environnement socioéconomique, sa " mise en politique », comme il est dit dans ces travaux. Ces constats permettent aux auteurs de préciser leur démarche conceptuelle et méthodologique, ce que nous étudierons dans un deuxième temps, fournissant ainsi les moyens de procéder à des comparaisons et de suggérer des suites à ces recherches. Ce double apport, empirique et théorique, conduit à renouveler - au moins à diversifier - nos regards sur les exploitations, la problématique de l'agriculture dans le développement et consécutivement sur les conceptions de politique agricole, ce que nous verrons dans un troisième temps. On l'aura compris, c'est la thèse de la nouveauté (et non de la rengaine, du retour ou de la diversion) qui sera défendue ici (voir encadré page suivante).

\section{L'agriculture familiale aujourd'hui}

On ne reviendra pas sur les acquis habituellement reconnus et même rabâchés concernant l'agriculture familiale : elle présente une plasticité qui lui fournit - souvent au prix de privations, d'efforts, de faibles rémunérations - une capacité (bien souvent, une obligation) de résistance aux difficultés et une faculté d'adaptation pour tenter d'en triompher (Malassis, 1958). La solidarité au sein de la famille, l'ardeur au travail, le courage de ses membres, etc., et surtout l'absence d'alternatives concourent à la mobilisation de tous. Ces caractéristiques habituellement soulignées sont souvent associées à des préférences idéologiques ou matérielles glorifiant le paysan, bon travailleur, bon chef de famille, bon citoyen, bon soldat... Sans nier la réalité de ces caractéristiques, les travaux examinés ici proposent des avancées sur un autre registre, celui de l'organisation et $\mathrm{du}$ fonctionnement de l'exploitation agricole familiale, laissant à d'autres le soin d'une valorisation artistique, 


\section{Encadré. Ouvrages publiés en 2014 et 2015 à l'occasion de l'année internationale de l'agriculture familiale,} analysés dans cet article ${ }^{1}$.

Acloque Desmulier D., Gasselin P., Rouillé d'Orfeuil H. (Eds), 2014. Agricultures familiales, trajectoires, modernités et controverses (I), Revue Tiers Monde, 220, 4.

Acloque Desmulier D., Gasselin P., Rouillé d'Orfeuil H. (Eds), 2015. Agricultures familiales, trajectoires, modernités et controverses (II), Revue Tiers Monde, 221, 1.

Bosc P.-M., Sourisseau J.-M., Bonnal P., Gasselin P., Valette É., Bélières J.-F. (Eds), 2015. Diversité des agricultures familiales. Exister, se transformer, devenir, Versailles, Quæ.

Gasselin P., Choisis J.-P., Petit S., Purseigle F., Zasser S. (Eds), 2014. L'agriculture en famille : travailler, réinventer, transmettre, Les Ulis, EDP Sciences, http:/ / www.edp-open.org/lagriculture-en-famille-travailler-reinventertransmettre.

Sourisseau J.-M., (Ed.) 2014. Agricultures familiales et mondes à venir, Versailles, Quæ.

Sourisseau J.-M., Kahane R., Fabre P., Hubert B. (Eds), 2015. Family farming \& research, Proceedings of international encounters, 1-3 June 2014, Montpellier, Paris, Agropolis International, http://www.agropolis.org/pdf/ publications/proceedings-international-encounters-family-farming-research-june-2014.pdf.

Thirion M.-C., Bosc P.-M. (Eds), 2014. Les agricultures familiales du monde. Définitions, contributions et politiques publiques, Montpellier/Paris, Cirad/Agence française de développement, http://www.afd.fr/webdav/site/afd/ shared/PUBLICATIONS/RECHERCHE/Scientifiques/A-savoir/28-A-Savoir.pdf.

1 [Le lecteur pourra également consulter le dossier $n^{\circ} 19$ d'Agropolis International consacré aux agricultures familiales : http:/ / www.agropolis.fr/pdf/publications/dossier-agricultures-familiales-janvier-2014.pdf, NDLR].

poétique, idéologique ou morale, ce qui n'exclut ni l'empathie ni même la bienveillance à l'égard du monde agricole.

La diversité d'opinions, de politiques, d'appréciations sur l'agriculture familiale, les interrogations qui en résultent sur sa signification et la justification d'une "Année internationale » qui lui est spécialement dédiée renvoient à des définitions multiples (Acloque Desmulier et al., 2014). Sans doute, toutes s'accordent pour souligner les liens entre la famille et l'exploitation : dans les exploitations agricoles familiales, le travail et le capital sont exclusivement ou principalement familiaux ; le terme " principalement » appelle la fixation de limites et donc une évaluation ouvrant la voie à des débats et des ambiguïtés, ou à des dénominations et dénombrements considérés comme légitimes par les uns, abusifs par les autres. Sans reprendre ici ces débats sur la définition de l'agriculture familiale, on signalera simplement que la question est très opportunément examinée dans ces travaux (Sourisseau, 2014 ; Thirion et Bosc, 2014 ; Acloque Desmulier et al., 2014) et que les auteurs se situent clairement dans le cadre d'une définition stricte qu'ils justifient en étudiant méticuleusement «l'état de l'art " : l'agriculture familiale « désigne des formes d'organisation de la production agricole caractérisées par (i) des liens organiques entre la famille et l'unité de production et (ii), par la mobilisation du travail familial excluant le salariat permanent. Ces liens organiques se matérialisent par l'inclusion du capital d'exploitation dans le patrimoine familial et par la combinaison de logiques [...] marchandes et non marchandes [...]» (Thirion et Bosc, 2014, p. 19). Ce choix strict présente l'avantage de la clarté et de la rigueur (dans le dénombrement et le raisonnement), il est situé par rapport aux alternatives proposées dans la littérature scientifique ou politique, il protège des dangers dus à la polysémie de l'intitulé de l'objet d'étude (Lamarche, 1992, 1994).

L'information internationale est évidemment lacunaire et hétéroclite, mais les ordres de grandeur peuvent être estimés et sont convaincants. " Les agricultures familiales représentent l'écrasante majorité des agricultures du monde avec un effectif se situant autour des 500 millions d'exploitations [...] Ce sont ces agricultures qui créent le plus d'emplois [...] Les exploitations à grande échelle avec recours au travail salarié [...] n'emploient que quelques millions du 1,3 milliard d'actifs agricoles mondiaux. » (Thirion et Bosc, 2014, p. 72). On peut dire, de façon imagée, que l'agriculture familiale est le $1^{\mathrm{er}}$ employeur mondial ! Elle fournit 60 à $70 \%$ de la production agricole mondiale (Rouillé d'Orfeuil, 2014). Que l'on examine les statistiques, les opinions, les politiques ou la vie socioéconomique, cette forme d'agriculture mérite donc quelques considérations.

\section{Le fonctionnement de l'exploitation agricole familiale}

Le constat le plus immédiat est d'abord celui de l'extension et de la permanence de cette forme d'exploitation. Permanence ne signifie pas identité figée, les auteurs résument souvent leurs pensées par des verbes 
indiquant le mouvement, le changement, la mobilité plus que la stabilité : dans les titres des ouvrages de Bosc et al. (2015) et Gasselin et al. (2014), on retrouve certes « exister», mais aussi « se transformer», « devenir», « réinventer », «transmettre », et les préoccupations d'avenir sont omniprésentes ( $c f$. 1'expression « mondes à venir » dans le titre du livre de Sourisseau [2014]).

Ce constat est dû à la polymorphie de ces exploitations. Celle-ci procède de l'invention de stratégies pour la survie, voire le mieux-être de la famille (diversification, émigration, multilocalisation, accès au crédit, valorisation d'actifs spécifiques...), et, sans doute, plus encore de rationalités diverses qui ne s'accommodent pas du monolithisme d'une rationalité technicoéconomique de l'entreprise et préfèrent considérer la sécurité (de l'alimentation, de l'emploi, du revenu...), le temps de travail, la résidence, les solidarités locales, les relations sociales... Serait-il inconvenant de considérer ces utilités non pas comme des futilités mais comme les premières préoccupations des familles? L'expérience montre qu'il existe une diversité d'options qui s'oppose à la vision habituelle d'une trajectoire prétendument unique, inéluctable, optimale... et évidemment bienfaisante ! Cela conduit nos auteurs à penser les exploitations agricoles familiales en termes de système (Acloque Desmulier et al., 2014), en approfondissant efficacement cette notion déjà proposée dans la littérature (au « Nord » comme au «Sud »). Cette approche a plusieurs conséquences.

D'une part, la diversité des terrains d'études permet de montrer les stratégies mises en œuvre et surtout leur logique alors que celles-ci sont souvent considérées comme archaïques, maladroites, inefficaces, voire irrationnelles. D'autre part, cette exigence scientifique de compréhension permet d'identifier les contributions (et limites) des exploitations agricoles familiales pour répondre aux défis d'aujourd'hui et demain (Sourisseau, 2014 ; Acloque Desmulier et al., 2015).

La bienveillance pour l'objet de recherche conduit parfois à un certain optimisme sur les qualités (innombrables !) de l'agriculture familiale (Acloque Desmulier et al., 2015) pour assurer toutes ces contributions. Cependant, les auteurs, réalistes et prudents, ne manquent pas de souligner que ces contributions possibles sont soumises à des conditions qui impliquent des actions collectives ou publiques. Tous nos auteurs parlent alors de « mise en politique » dégageant ainsi une thématique à la fois nouvelle et essentielle à la compréhension et à la gestion des agricultures familiales.

\section{La « mise en politique » des agricultures familiales}

L'agriculture familiale n'est pas seulement une collection d'exploitations agricoles familiales, c'est un ensemble plus large impliquant des institutions leur permettant de fonctionner en satisfaisant les contributions attendues (approvisionnement alimentaire, performances agroalimentaires, organisation de l'amont et de l'aval, emploi, participation à la vie collective...). De manière générale, cet environnement institutionnel concourt à la reconnaissance (économique, juridique, citoyenne) des agriculteurs familiaux et à la sécurisation de leur statut (indispensable à la prise de décision toujours risquée).Toutes ces organisations entourant progressivement les exploitations agricoles familiales supposent (i) des formules juridiques pour répondre à leurs besoins et les articuler avec les attentes de la société, (ii) des incitations en faveur de la création, l'animation, l'évaluation de ces institutions.

À cet égard, les auteurs dégagent de leurs nombreuses études de terrain (Bosc et al., 2015) un « enseignement majeur » (Sourisseau, 2014 ; Acloque Desmulier et al., 2014) : la disjonction des politiques et des instruments - entendons par là le manque de cohérence, d'affinité, d'adaptation entre les politiques publiques habituelles et la réalité de l'agriculture familiale. Ces observations sont souvent renforcées par la disjonction des politiques publiques qui restent largement sectorielles (politique agricole, environnementale, sociale, de formation...). En schématisant, les politiques et leurs instruments sont orientés vers une modernisation agricole selon une vision sectorielle (avec une activité strictement définie et délimitée, l'offre et la demande de biens agricoles, le rôle des prix comme signaux efficaces de sélection...), tandis que les exploitants familiaux recherchent d'abord la survie de leur famille et son bien-être. Dans de très nombreux pays en voie de développement, la masse de travailleurs agricoles et ruraux, la faible création d'emplois non agricoles, les obstacles à la mobilité intersectorielle ne peuvent pas laisser espérer une modernisation aussi rapide que celle qu'ont connue les pays aujourd'hui industrialisés. Doit-on alors s'étonner du manque de congruence entre ces deux visions? On est bien invité, comme le proposent ces travaux, à repenser l'action publique : on voit l'ampleur du travail (Acloque Desmulier et al., 2014)!

Cette «mise en politique » doit donc concourir à la réunion des conditions de fonctionnement de l'agriculture familiale : sécurité foncière, améliorations techniques, approvisionnement, infrastructures matérielles, conditions commerciales, juridiques et financières des échanges. La mobilité intersectorielle du travail doit aussi être envisagée : les exploitants, qui ne pourront pas ou ne voudront pas participer aux améliorations proposées, seront, eux et/ou leurs enfants, progressivement éliminés et devront rechercher du travail. Certes, au regard de l'emploi, l'agriculture familiale est plus inclusive que d'autres formes de production mais il est illusoire de compter sur elle pour résorber efficacement le 
sous-emploi agricole plus ou moins caché. On se trouve en présence d'un ensemble complexe opportunément affirmé ici, sans pouvoir être détaillé mais qui suggère des voies de recherche pour l'avenir. Cet apport de la littérature examinée dans le présent article évite de laisser croire que ces transformations seront spontanées ou automatiques : les améliorations attendues des contributions de l'agriculture familiale au développement socioéconomique supposent généralement des changements dans l'organisation agraire en place, qui réclament un accompagnement attentif.

Faute de cet accompagnement, non seulement les attentes ne seront pas satisfaites mais, de plus, la situation de la paysannerie stagnera ou même périclitera, décevant les espoirs mis dans l'agriculture familiale et démobilisant les efforts engagés. Les modalités de cette « mise en politique » sont donc loin d'être secondaires ou accessoires, elles doivent conforter l'organisation familiale et améliorer ses performances économiques, sociales et environnementales. Les expériences historiques de transformation de système agraire montrent la diversité des choix qui, selon les circonstances, s'offrent pour organiser cette "mise en politique ». Hormis l'appel pressant et systématique en faveur de la participation des populations aux décisions les concernant, ce domaine de préoccupations mérite des approfondissements ; une illustration en est utilement fournie dans la réflexion sur les relations entre l'agriculture familiale et la recherche (Sourisseau et al., 2015).

On passe ainsi de la considération des exploitations aux questions concernant le modèle de développement. Cette abondante documentation relative à de nombreux terrains (Bosc et al., 2015) amène les auteurs, soucieux, à juste titre, de ne pas en rester à une accumulation factuelle inévitablement hétéroclite, à préciser opportunément leur démarche conceptuelle et méthodologique, de manière à dégager des enseignements génériques et capitalisables permettant de proposer des méthodes d'approche de nouvelles situations ou de procéder à des comparaisons.

\section{Acquis méthodologiques et conceptuels}

À des fins de clarification, on distinguera les approches consacrées à l'organisation des exploitations agricoles familiales et celles consacrées à leur fonctionnement et à leur insertion dans l'économie englobante. Les premières ne sont pas entièrement spécifiques à l'agriculture familiale et sont aussi utiles pour aborder $\mathrm{d}^{\prime}$ autres formes d'organisations agraires, comme les auteurs en font la preuve à l'occasion (Bosc et al., 2015 ; Sourisseau, 2014 ; Acloque Desmulier et al., 2015). Les secondes, soulignées avec force par les réflexions sur la « mise en politique » de l'agriculture familiale, sont encore en construction et justifieront certainement de nouvelles recherches dans l'avenir.

\section{Sur l'organisation des exploitations agricoles familiales}

Cette série d'ouvrages, réalisés par une vingtaine de chercheurs à partir de recherches de longue durée, présente, outre une identité d'objet, une proximité quant à la posture intellectuelle qui leur permet de dégager des conclusions méthodologiques, sans en rester, comme c'est souvent le cas, aux comptes rendus des cas étudiés. Au moment où la place des sciences sociales, notamment de l'économie, est débattue et où on s'interroge sur leur utilité, leur impact et les relations entre elles, il paraît important de noter les principales caractéristiques de la posture retenue ici, qui s'inscrit dans une démarche initiée par des recherches "tropicalistes », devenues aujourd'hui « anciennes » (AMIRA, 1978 ; Benoit-Cattin et Faye, 1982 ; Gastellu, 1980).

Ce qui apparaît au premier abord, c'est la dimension mondiale du propos. Cette dernière offre bien sûr une diversité de cas étudiés, mais invite aussi à leur comparaison, rendue possible par un accord sur l'objet, la problématique et les objectifs (Sourisseau, 2014 ; Bosc et al., 2015).

Cet élargissement mondial est d'autant plus notable qu'il s'accompagne toujours d'une connaissance intime de la réalité étudiée. Cette familiarité avec le terrain offre des occasions de découvertes originales (ici, par exemple, la pluriactivité, la multifonctionnalité, l'organisation foncière, la vie familiale, les lieux de décision...). Cette intimité débouche sur une posture compréhensive : le constat de diversité, banal s'il s'agit simplement d'une photographie ou d'un résultat statistique, devient innovateur et tonique si les constituants de cette diversité sont expliqués (plus sérieusement que sur le mode de la marginalité, de l'exception, de la « niche »). C'est évidemment postuler une logique à découvrir et à révéler, c'est refuser l'ignorance ou le dogmatisme souvent inhérent à un modèle unique et impératif, qui limite le champ de pensée de l'observateur.

Ainsi, le lecteur passe-t-il de la seule considération $\mathrm{d}^{\prime}$ une fonction de production à celle du cadre social et institutionnel de l'unité de production. Cet élargissement de l'analyse conduit à examiner le système d'activité de l'exploitation agricole familiale, sa genèse, sa gestion par des familles, dont on sait bien qu'elles se décomposent et se recomposent (Segalen, 2012 ; Rémy, 2010). Ce système peut être compris en étudiant les rapports sociaux, internes et externes, qu'il entretient : les rapports internes sont constitutifs de son organisation (rapports entre générations, entre sexes, place dans la production, dans la prise des décisions...) ; les rapports externes dépendent des relations des familles avec leur 
environnement socioéconomique (les règles coutumières, les autres exploitations, les institutions agricoles, les emplois et revenus extérieurs...). La prise en compte de ces rapports permet généralement de mieux expliquer et d'anticiper la dynamique, plus ou moins conflictuelle, des exploitations agricoles familiales diversifiées. La " mise en politique ", présentée ci-dessus parmi les constats novateurs de ces recherches, trouve, dans ces rapports internes et externes, des fondements intellectuels.

Cet élargissement de l'analyse, dont on vient de souligner plusieurs aspects (géographique, social, organisationnel), est aussi prospectif : il confronte l'exploitation agricole familiale aux défis du monde de demain : sécurité alimentaire et sanitaire, pauvreté, technologie, environnement (Sourisseau, 2014 ; Acloque Desmulier et al., 2015).

L'élargissement concerne enfin le mode d'approche qui ne se limite pas à étudier la fonction de production comme l'ingénieur, ou l'entreprise comme l'économiste et le gestionnaire, ou le régime de propriété comme le juriste, ou l'organisation familiale comme le sociologue, mais qui tente de combiner ces démarches pour rendre compte de l'organisation du binôme exploitationfamille. Les disciplines scientifiques sont mobilisées non pas dans une perspective fusionnelle ou totalisante mais sur le mode de la complémentarité et de la coopération appelées par l'identité de l'objet. L'économiste tient compte des apports du socioanthropologue (par exemple, à propos du régime foncier, des instances de décision...) qui, lui, a intérêt à connaître la formation et l'utilisation des revenus ou encore les pratiques techniques étudiées par l'agronome en fonction des techniques disponibles (et accessibles), des caractéristiques du système productif et des incitations prodiguées ou envisagées par les politiques publiques. L'habituel cloisonnement des disciplines fait place à leur dialogue et stimule leur progression. Cette analyse de la démarche pratiquée dans les ouvrages étudiés n'est évidemment pas spontanée ou immédiate, elle suppose un important travail de coconstruction et la recherche de compromis entre les participants.

\section{L'intégration des exploitations agricoles familiales dans l'économie et la société}

Cette notion de système d'activités ouvre la voie à l'étude des relations des exploitations agricoles familiales avec leur environnement : relations avec l'amont et l'aval mais aussi emplois et revenus extérieurs, accès à l'information, aux connaissances, participation aux organisations paysannes. Ces relations, marchandes ou non, sont opportunément soulignées par nos auteurs (Bosc et al., 2015), compensant ainsi l'oubli, l'ignorance, voire le mépris fréquent des relations non marchandes par les politiques publiques et même parfois par la recherche.
Mais le paysage est peut-être en cours de changement : certes, la politique publique est toujours dominée par le slogan « market oriented » mais devient plus attentive aux biens publics et biens communs, aux relations biens marchands/biens non marchands (Parance et de Saint Victor, 2014).

Il s'agit d'abord de remédier aux cas classiques de «défaillances de marchés », telles que les conçoivent les économistes standards : externalités et biens publics qui sont justement nécessaires au fonctionnement d'une agriculture familiale et qui justifient une régulation publique. De même, des coûts de transaction élevés peuvent conduire à préférer une régulation par une organisation, un système hiérarchique plutôt que par le marché. L'aversion aux risques générés par le fonctionnement des marchés nuit à l'efficacité du mécanisme des prix. L'inégalité (fréquente) des acteurs entraîne des structures de marchés dans lesquelles les prix ne sont pas de bons indicateurs des préférences des consommateurs et des performances des producteurs. Un « bon » fonctionnement du marché lui-même implique une organisation institutionnelle qui n'est généralement pas d'essence marchande.

Toutes ces limites au marché (rapidement rappelées ici) légitiment l'attention portée à l'organisation, à la coopération, à la négociation. C'est particulièrement le cas des agricultures familiales, plus encore que les situations d'agriculture patronale (entrepreneuriale) où la concurrence (éventuellement aménagée) constitue le mécanisme régulateur principal. C'est pourquoi les travaux examinés ici sont particulièrement attentifs à l'action collective, aux biens publics, à la création de biens communs (Thirion et Bosc, 2014 ; Bosc et al., 2015). Cette problématique explique une considération renouvelée, notamment en faveur :

- du territoire, non pas d'abord comme support d'activités mais comme lieu d'organisation, de création, d'innovation ;

- de l'action collective, de son contenu marchand et non marchand («matériel et idéel »), qui ne sont certes pas nouveaux (via les associations, coopératives, mutuelles, syndicats...), mais dont on reconnaît aujourd'hui l'importance économique, sociale, politique ;

- de l'innovation, en insistant ici sur sa dimension sociale. On s'intéresse à la conception d'innovation adaptée aux circonstances locales plutôt qu'à l'innovation standardisée, normalisée, prétendument " passepartout».

Cet ensemble de travaux fait une place à quelques analyses d'agriculture non familiale (Europe, Amérique du Sud) (Sourisseau, 2014 ; Thirion et Bosc, 2014 ; Acloque Desmulier et al., 2014). Il ne faut pas voir là une dérive par rapport à une stricte définition de l'objet de ces publications, mais une complémentarité bienvenue, dont il faut souhaiter la poursuite (Acloque Desmulier 
et al., 2015). On dispose ainsi d'informations permettant des comparaisons entre diverses formes sociales de production, notamment entre les exploitations agricoles familiales et les exploitations patronales. Cependant, ces comparaisons sont finalement peu pratiquées dans ces travaux, alors qu'elles pourraient avantageusement éclairer les conditions de concurrence entre ces différentes formes de production. Cette concurrence débouche-t-elle sur une différenciation durable ou sur le succès de l'une d'entre elles, éliminant ou recomposant les autres ? L'analyse des trajectoires d'une forme d'organisation à une autre éclairerait opportunément la dynamique du système productif. La question est d'autant plus importante que les politiques agricoles se sont souvent donné pour objectif de faciliter la trajectoire faisant passer de l'exploitation agricole familiale à l'exploitation " entrepreneuriale » ou "patronale » (selon la terminologie retenue dans ces travaux), que l'on considère souvent comme encore familiale ou quasi familiale car le salariat y reste très modeste. De plus, les formes hybrides (Acloque Desmulier et al., 2015), associant main-d'œuvre familiale et salariée, sont fréquentes en agriculture comme dans d'autres secteurs économiques avec lesquels des comparaisons pourraient être utiles. Nos auteurs parlent alors de « l'organisation en famille» (Gasselin et al., 2014) pour rendre compte de l'éloignement croissant des familles et des exploitations (par les activités, les revenus, les unions conjugales, les résidences, les formes juridiques, les installations...) sans, pour autant, qu'il y ait séparation totale des deux entités (entraide, financement, propriété, logement, gestion unifiée des revenus...).

Cette démarche d'analyse, mise à l'épreuve sur de nombreux terrains (Bosc et al., 2015), permet des comparaisons internationales.

\section{Comparaisons internationales}

Les travaux dont il est question dans le présent article permettent à leurs auteurs de proposer une méthode pour analyser les formes d'agriculture familiale : définition de l'unité pertinente d'observation, recherche compréhensive des logiques d'acteurs (prise de décision, pratiques, mises en œuvre), organisation et évolution, mode d'insertion économique, sociale, politique. Deux thèmes de cette démarche, relevés ci-dessus comme porteurs d'avancées bienvenues, les systèmes d'activités et « la mise en politique », facilitent la comparaison avec le modèle SRL («Sustainable Rural Livelihoods ») (Chambers et Conway, 1991 ; Scott, 1976 ; Small, 2007), dont on connaît l'audience et l'impact dans les recherches et la pensée internationales contemporaines. Les travaux dont nous parlons ici y sont attentifs.
Ainsi, la démarche de ces travaux présente des proximités évidentes (recherche de l'unité pertinente, attention aux acteurs, améliorations des performances) avec le modèle SRL, mais aussi des différences qui peuvent offrir des occasions de débats et d'améliorations. On notera en particulier :

- la considération des relations intrafamiliales (hommes/ femmes, jeunes/anciens, autoconsommation, trésorerie, épargne...), bien utiles à la compréhension des décisions, de la répartition des activités..., qui est particulièrement étudiée dans les ouvrages examinés dans le présent article ;

- l'évaluation des capitaux mobilisés qui se fait, dans le modèle SRL, en référence aux marchés, aux substitutions possibles entre facteurs de production ; les données marchandes sont ainsi privilégiées au détriment de l'intangible. Mais, toutes les ressources ont-elles un prix monétaire ? C'est ignorer les externalités et biens publics qui, justement, retiennent l'attention de nos auteurs ;

- les relations avec le contexte économique, social, politique, dont on a rappelé l'importance ci-dessus avec la « mise en politique », qui ne sont qu'exceptionnellement sous la dépendance unique du marché. Même les relations économiques les plus banales procèdent à la fois de marchés et $d$ 'organisations.

En résumé, dans les démarches et propositions présentées dans ces ouvrages, les données non marchandes, le « matériel » et l'« idéel » (Godelier, 1984), les « capabilités » (Sen, 1999) concourent à l'analyse et à la gestion des différentes formes d'agricultures familiales.

Le rapprochement des agricultures familiales des pays en voie de développement et des pays développés (Gasselin et al., 2014 ; Acloque Desmulier et al., 2014) peut surprendre, tant les différences sont importantes. Il s'avère pourtant bénéfique ; il permet d'identifier des questions communes (par exemple, les relations intrafamiliales, les technologies adaptées, l'organisation de l'encadrement commercial, juridique, politique des unités productives...) et de comparer les réponses fournies (ou envisagées) selon les circonstances locales, notamment les technologies accessibles et les règles sociojuridiques (régissant le fonctionnement des échanges). On peut d'ailleurs remarquer que ces proximités constatées dans l'organisation et le fonctionnement de l'agriculture familiale des pays en développement et des pays développés se confirment dans les interprétations proposées. Depuis les années 1960, on s'intéresse aux revenus non agricoles des ménages agricoles, ce qui a conduit, dans les années 1970, à considérer, dans différents pays, la notion de système d'activité (Paul et al., 1994). Un nouvel intérêt partagé est advenu ensuite avec l'attention portée aux organisations paysannes, constitutives de ce qui est appelé ici «mise en politique » ou, ailleurs, appareil de développement. Enfin, aujourd'hui, le rôle du territoire 
est valorisé dans les situations les plus diverses (Thirion et Bosc, 2014 ; Aubert et al., 2009). L'agriculture familiale en pays développés fournit une nouvelle illustration des capacités d'adaptation des exploitations familiales qui ne sont pas l'apanage des seuls pays en voie de développement.

\section{De nouveaux regards?}

Autant par leurs apports empiriques que par leurs interprétations conceptuelles, ces ouvrages suggèrent un renouvellement des regards sur l'agriculture et ses transformations structurelles à long terme; ce n'est pas la moindre de leurs contributions à la pensée contemporaine du système productif agricole, de sa place dans la société et de sa gestion. C'est pourquoi ces suggestions de renouvellement méritent une attention particulière qui dépasse le seul intérêt de l'agriculture familiale.

\section{La place de l'agriculture dans l'économie et la société}

Selon l'analyse canonique, la place de l'agriculture est pensée à travers les mécanismes élémentaires de l'ajustement concurrentiel marchand : les gains de productivité acquis par la modernisation, l'inélasticité de la demande alimentaire, les incitations fournies par les prix concourent au transfert de ressources engagées dans l'agriculture, notamment du travail, vers les secteurs non agricoles. C'est ce qui expliquerait la modernisation agricole et la croissance d'une économie diversifiée. Cette vision optimiste est souvent temporisée par le coût et la lenteur de ces mécanismes qui légitiment une intervention publique. Les conditions de ce modèle sont assez restrictives, le rythme de création des emplois susceptibles d'accueillir les agriculteurs libérés est sans commune mesure avec les effectifs concernés (Badouin, 1967 ; Malassis, 1973 ; Hayami et Ruttan, 1998 ; Jacquet et al., 2012 ; Rouillé d'Orfeuil, 2013). Les mobilités attendues rencontrent de nombreux obstacles. La modernisation sectorielle, qui a effectivement amélioré la sécurité alimentaire, a engendré de multiples problèmes, largement soulignés aujourd'hui (environnement, qualité, crises, coûts...). D'où l'idée de rechercher une alternative - ou, plus modestement, un complément - à cette analyse habituelle, qui pourrait venir de l'agriculture familiale grâce à ses caractéristiques et potentialités.

La sensibilité de ces travaux, très attentifs à la diversité et réservés sur les analyses unidimensionnelles ou monofactorielles, accentue évidemment le caractère mécanique des études qui débouchent sur des évolutions obligées, quasiment standardisées, ignorant ou sous-estimant les spécificités locales, la complexité originale des situations, les circonstances handicapant plus ou moins le déroulement de ces mécanismes. La force de cette critique - qui demande encore du travail ! - vient des recherches présentées, de leurs fondements empiriques, de leur interprétation théorique. Sans revenir sur les caractéristiques et le fonctionnement des exploitations agricoles familiales et des autres formes de production, on peut, en schématisant, distinguer deux idéauxtypes appelés par les auteurs « exploitation sectorielle » et « exploitation territoriale » (Gasselin et al., 2014).

L'exploitation sectorielle est pensée à partir des caractéristiques économiques du secteur agricole : l'offre, la demande, les prix. C'est le modèle classique proposé aussi bien par l'analyse économique courante (Samuelson, 1967) que par les politiques agricoles qui généralement s'en inspirent. L'exploitation modernisée triomphe dans la concurrence marchande, les autres sont éliminées. On a vu que, de manière souvent inattendue et contradictoire avec ce premier modèle, l'exploitation agricole familiale n'épousait pas cet idéal-type et s'organisait autour de la famille pour satisfaire ses besoins. C'est dans la localité que les stratégies familiales sont conçues et mises en œuvre à travers des relations marchandes et non marchandes. On peut ainsi parler d'exploitation « territoriale » dans la mesure où elle est pensée à partir des familles, des activités, des revenus, en relation avec l'environnement naturel, social, politique.

Dans le premier cas, on regarde l'entreprise (en cherchant à la séparer de plus en plus nettement de la famille), son organisation, ses performances. Dans le second, on s'intéresse à la famille, ses activités, ses relations internes et externes. L'exploitation sectorielle est essentiellement insérée dans les filières agroalimentaires ; l'exploitation territoriale est intégrée dans une localité. La modernisation "classique » est une artificialisation standardisée, affranchie du territoire, excluant les agriculteurs non compétitifs. L'évolution des exploitations territoriales mobilise des biens publics, des diversifications créatrices d'emploi, des initiatives qui ne sont pas systématiquement exclusives mais, au contraire, souvent inclusives (Gasselin et al., 2014 ; Bosc et al., 2015). Certes, cette présentation d'un renouvellement $d u$ regard sur les unités de production et le secteur agricole est succincte mais le lecteur intéressé trouvera un argumentaire plus serré et nuancé dans les ouvrages analysés. Ce renouvellement des idées se retrouve également à des niveaux plus globaux, comme celui de la politique agricole.

\section{La politique agricole}

Tous les travaux étudiés soulignent avec force l'importance des politiques publiques sans, pour autant, que cette question constitue le cœur des ouvrages. On en reste donc à des principes et des ambitions sans détailler les mesures, les instruments, les conditions de mise en 
œuvre. Ces dernières préoccupations appellent de nouvelles recherches.

Les propositions avancées se différencient, souvent s'opposent. Les politiques habituellement préconisées cherchent à moderniser le système productif, elles font confiance au marché en suggérant parfois des aménagements (par exemple, organisation de l'offre pour améliorer le pouvoir de négociation des agriculteurs, diffusion d'informations, voire régularisation des cours...). On connaît les critiques formulées à l'encontre de ce type de politique : sélectivité, inégalité, dépendance, inadaptation aux défis d'aujourd'hui et de demain, en termes de pauvreté, sécurité alimentaire et gestion des ressources naturelles. Face à ces insatisfactions, une alternative (tout du moins en partie), fondée sur les exploitations agricoles familiales, largement dominantes dans le monde, serait de valoriser leurs fonctions, de réunir les conditions, marchandes et non marchandes, de leur système d'activités, d'assurer leur " mise en politique ». Pour cela, la politique agricole devrait être étendue à une politique rurale ou territoriale (Thirion et Bosc, 2014). $C^{\prime}$ est en effet à ce niveau que se forment les conditions de fonctionnement de l'exploitation agricole familiale et de 1'accomplissement de ses potentialités. Cette politique alternative suppose une reconnaissance économique, sociale, politique des exploitants familiaux et leur participation à la gestion des affaires agricoles et rurales. Un prolongement de ces propositions très générales pourrait concerner différents domaines d'intervention; ces ouvrages suggèrent notamment trois pistes d'approfondissement :

- la sécurisation des agricultures familiales, notamment l'organisation de l'accès à la terre (Le Roy et al., 1996), la régularisation des cours (Boussard, 2007 ; Galtier, 2012), la facilitation des échanges (infrastructures matérielles, outils juridiques) ;

- l'accompagnement des systèmes d'activités diversifiées (création d'emplois, prise en compte de l'environnement, conception d'innovations techniques et sociales, droit du travail, formation...) ;

- la mise à disposition, pour les agriculteurs, d'outils de participation responsable à l'organisation de la vie économique, sociale, politique.

Les différences avec la vision traditionnelle dominante (mais obsolète, selon la professeure Friedmann dans sa préface de l'ouvrage de Bosc et al. [2015]) sont claires. La préoccupation principale n'est pas la même : une modernisation technicoéconomique sélective dans un cas, l'accompagnement d'un milieu socioéconomique inclusif dans l'autre (que les auteurs des ouvrages examinés décrivent par les qualificatifs suivants : "politiques publiques ciblées, modulables, multidimensionnelles et inclusives ») (Thirion et Bosc, 2014). La mise en œuvre est aussi soumise à des procédures différentes : la politique habituelle est dans le champ de la prescription et de l'exécution de modèles standardisés, définis ailleurs (dans les organismes de recherche, les firmes agroalimentaires ou l'administration) ; la politique alternative fait une place à la conception, à l'invention, à l'évaluation des projets par les acteurs. On retrouve alors le territoire comme lieu d'innovation, que l'on peut espérer adaptée à la diversité des situations locales. Il s'agit ici d'une politique d'accompagnement, dotée de capacités d'incitations, qui associera marchés et organisations, relations marchandes et non marchandes.

\section{Et demain ?}

Ces travaux, par l'accent mis sur la diversité de l'agriculture familiale et, a fortiori, de l'agriculture dans son ensemble, et par leurs propositions d'un renouvellement des problématiques, invitent à se poser la question de l'avenir à long terme. Après avoir approfondi les contributions de l'agriculture familiale aux défis d'aujourd'hui et demain, souligné le besoin d'une politique adaptée, nos auteurs suggèrent rapidement des scénarios prospectifs; ainsi, dans la conclusion de Thirion et Bosc (2014), quatre scénarios sont envisagés :

- maintien de l'agriculture familiale ;

- transformation de l'agriculture familiale en agriculture « patronale » ou « entrepreneuriale »;

- disparition de l'agriculture familiale ;

- renforcement de l'agriculture familiale.

Ces scénarios restent limités à l'agriculture familiale, sans analyse approfondie des formes d'organisation susceptibles de la concurrencer, de la transformer, voire de l'éliminer. Les trajectoires de passage de l'agriculture familiale à d'autres formes sont peu abordées ; il est vrai que la définition stricte de l'objet d'étude (rappelée au début de cet article) n'incite pas à considérer activement ces autres organisations. Il en résulte que la dynamique de ces scénarios se concentre sur le maintien, la reproduction de l'agriculture familiale, alors que, dans le passé récent, on s'interrogeait sur la stabilité et la durabilité des exploitations agricoles familiales, notamment celles pluriactives.

Certes, les arguments en faveur de leur pérennité apparaissent convaincants. La démographie en est une des raisons : la masse d'agriculteurs familiaux ne peut pas être rapidement absorbée par les secteurs non agricoles qui ne créent pas suffisamment d'emplois, la mobilité intersectorielle est lente et coûteuse, la pression démographique reste forte. Le fonctionnement économique des unités de production contribue également à leur pérennité : les capacités de résistance, d'adaptation et d'innovation, amplement soulignées, comme les difficultés d'accès aux formes non familiales ou à une modernisation sélective sont des facteurs qui n'incitent pas au changement. 
Dans de nombreuses sociétés contemporaines, les familles se réorganisent (Rémy, 2010) et, à cette occasion, renouvellent leur relation avec l'agriculture. C'est sans doute le sens du titre de l'ouvrage de Gasselin et al. (2014), L'agriculture en famille, plus spécialement consacré à la situation de l'agriculture familiale dans ces sociétés. La stricte correspondance de l'exploitation et de la famille a incité à une vision fusionnelle de ces deux entités; le modèle de Chayanov en est une illustration comme, sur le plan des politiques publiques, les lois françaises des années 1960 affirmant leur préférence pour l'exploitation familiale à 2 unités de travail humain (UTH). Les systèmes d'activités diversifiés au sein des ménages, les stratégies « hors cadre familial », l'orientation professionnelle des enfants, le choix des conjoints (Giraud et Rémy, 2008), les formes sociétaires... témoignent d'un relâchement des liens exploitations/familles. On a pu dire que les familles agricoles se "désagricolisaient » et Rémy (2010), en approfondissant ces constats, pose la question d'une agriculture post-familiale. On voit même des familles bien dotées en capital social et culturel qui acquièrent une activité extérieure et délèguent les travaux agricoles à des tiers (Benjamin, 1996 ; Aubert et Perrier-Cornet, 2009). Simultanément, l'organisation des exploitations tend à s'affranchir, au moins partiellement, des familles, à travers des coopérations interentreprises (entraide, spécialisation, assolement en commun, sociétés...), la délégation de travaux, la diversification, la transmission "hors famille » (Purseigle, 2015 ; Nicourt, 2013)... Le processus de modernisation des exploitations agricoles familiales (améliorations des performances, croissance du capital engagé, insertion dans les échanges) appelle ces transformations.

La vision canonique de l'exploitation reposait sur une association qu'on pouvait représenter par l'identité : 1 exploitation $=1$ famille $=1$ revenu agricole devant financer la consommation et l'investissement. Aujourd'hui, l'unité productive prend souvent l'allure d'un puzzle diversifié (d'activités, de ressources, de revenus, de résidences, de productions de biens et services) et évolutif. On parle parfois d' « exploitation flexible » (Séronie et Boullet, 2007).

Cette réorganisation notable des relations exploitation/famille ne semble pas déboucher rapidement sur une rupture radicale séparant complètement et définitivement ces deux pôles de l'agriculture familiale. Elle modifie le regard sur l'exploitation agricole familiale et invite à élargir sa définition acceptant une éventuelle différenciation des facteurs de production: présence de salariés s'ajoutant à la main-d'œuvre familiale, multiplicité des propriétaires fonciers. Le capital engagé reste habituellement familial ; même les formes sociétaires, parfois évoquées comme un indicateur d'une séparation exploitation/famille, sont précisément pratiquées pour pérenniser le cadre familial. Les candidats au métier d'agriculteur qui, on le sait, sont nombreux, ont un projet de travail indépendant et sont souvent mal dotés en capital. Rares sont les travaux réalisés sur les exploitations qui imposent une concentration de main-d'œuvre et, quand c'est le cas, divers types de coopération peuvent s'en charger. La concurrence entre les formes de production est souvent favorable à l'agriculture familiale qui, faute d'alternative, continue à produire, même si ses facteurs de production ne sont pas rémunérés aux taux des marchés, réduisant ainsi le coût d'approvisionnement pour le secteur agroalimentaire d'aval et pour les consommateurs. Bref, comme on l'a déjà souligné, l'agriculture familiale est compatible avec des évolutions diverses, des organisations plurielles, des statuts multiples.

Ces exploitations familiales et « modernes » ont élargi leur taille, sont insérées dans les échanges, les indicateurs économiques guident leurs décisions d'activités et $\mathrm{d}^{\prime}$ emplois. Elles se réclament d'ailleurs volontiers du modèle de l'entreprise (Barthélémy, 1988) en se comparant aux activités artisanales, commerciales ou de services avec lesquelles elles partagent de nombreuses caractéristiques (le capital est familial, le travail associe des travailleurs familiaux et salariés, l'insertion dans les marchés est acquise, la responsabilité reste personnelle).

Ces considérations expliquent aussi pourquoi les grandes exploitations «industrielles », capitalistes ou socialistes, n'ont pas connu, jusqu'à présent, le succès, pourtant souvent annoncé. Certes, il existe des situations d'agriculture non familiale pour lesquelles il n'y a pas de liens familiaux ni au sein du capital, ni parmi les travailleurs, ni entre travail et capital. La rupture exploitation/famille est ici complète, un capital anonyme devient l'instance principale de décision. Mais ces situations procèdent généralement de circonstances particulières : décision politique autoritaire en faveur d'une organisation "supérieure », placement de capitaux à la recherche d'opportunités de profit (cas de produits de luxe plus ou moins contingentés, de rentes d'innovateurs...), main-d'œuvre disponible, accès facile à la terre, recherche d'une garantie d'approvisionnement en produits indispensables (agriculture de plantation, recherche d'une sécurité alimentaire, conquête de nouveaux espaces, legs historiques d'une stratification sociale traditionnelle...).

Ces entreprises affranchies de relations étroites avec une famille espèrent bénéficier, grâce à leur taille et à leur performance, d'une compétitivité supérieure à celle des exploitations agricoles familiales, mais les coûts des premières sont largement contraints, incontournables tandis que les secondes, dans des stratégies d'association ou encore dans leur capacité de résistance, peuvent trouver des compensations à la faiblesse de leurs ressources. Ces grandes exploitations « industrielles » ne sont pas l'objet des recherches examinées ici, mais elles peuvent 
entretenir des relations (qui ne sont pas neutres) avec l'agriculture familiale : conflits, coopérations, reconnaissance, emploi... Selon ces perspectives, l'évolution des unités productives s'expliquerait alors par le mode de combinaison entre les recompositions des familles, d'une part, et les réorganisations des exploitations, $\mathrm{d}^{\prime}$ autre part, ou - si on accepte un langage jargonneux mais peut-être stimulant - entre la "désagricolisation » des familles et la "défamilialisation » des exploitations.

En s'appuyant sur les matériaux fournis par les ouvrages analysés ici mais aussi sur les connaissances disponibles concernant l'évolution des structures agricoles, on peut suggérer cette hypothèse de travail pour aborder la dynamique des exploitations aujourd'hui. Formulée de manière plus générale, on peut dire que l'agriculture familiale nous invite à analyser les rapports entre la sphère domestique et la sphère économique : fusionnées dans l'exploitation agricole familiale traditionnelle, ces sphères s'organisent et se renouvellent plus indépendamment aujourd'hui. Ce renouvellement concerne les relations (marchandes et non marchandes) internes (à la famille et à l'exploitation) et externes (avec l'environnement), ce qui nous fait retrouver les thèmes $\mathrm{du}$ 《territoire » et de la « mise en politique », souvent évoqués ci-dessus. L'analyse de l'organisation du système productif agricole trouve ainsi une actualité scientifique qui s'ajoute à son actualité politique... Le projet $\mathrm{d}^{\prime}$ " Observatoire des agricultures du monde », davantage réclamé en période de crise qu'en période de calme, pourrait opportunément éclairer ces actualités.

\section{Conclusion}

Au terme de cette lecture commentée d'une série d'ouvrages scientifiques consacrés à l'agriculture familiale, plusieurs constats de nouveautés se dégagent sur cette organisation pourtant ancienne et généralisée. Rappelons d'abord les plus évidents : une vision mondiale, une confrontation de l'agriculture familiale aux défis lancés à l'agriculture d'aujourd'hui et de demain. Mais, plus fondamentalement, la compréhension de l'agriculture familiale progresse grâce à l'analyse de son fonctionnement interne et surtout grâce à la conception d'un environnement sociopolitique adapté, permettant sa «mise en politique ». Ces nouveautés empêchent de considérer ces publications comme un simple rappel des connaissances disponibles ou, pire, comme une rengaine des discours les plus éprouvés. Elles invitent, au contraire, à un renouvellement de l'analyse des exploitations et des transformations structurelles de l'agriculture.

La nouveauté se constate aussi dans les recherches futures que ces travaux semblent appeler. On a signalé ci-dessus l'intérêt de considérer les rapports de l'agriculture familiale avec les autres formes d'organisation. On pourrait sans doute ainsi s'exprimer plus clairement sur la dynamique des systèmes productifs. Si les arguments en faveur d'une "mise en politique » apparaissent convaincants et bienvenus, les modalités de son organisation, de sa mise en œuvre pourraient constituer une suite logique et utile. L'attention parfois enthousiaste portée à l'agriculture familiale valorise sa capacité d'adaptation mais le coût de cette capacité, la nature des bénéficiaires et des perdants sont peu considérés. Or, il ne suffit pas de parler de politique « inclusive », encore faut-il la concevoir, l'organiser, la gérer, l'évaluer (Gasselin et al. 2014 ; Acloque Desmulier et al., 2015).

Ces travaux conduisent à un dernier commentaire sur l'organisation de ces recherches, à l'attention notamment des non-spécialistes et des administrations gestionnaires. On ne peut qu'être frappé par la concordance de l'actualité sociopolitique : l'ONU qui, comme on l'a dit, a décrété 2014 «Année internationale de l'agriculture familiale » et la parution de cette série d'ouvrages scientifiques. Cette fois-ci, la science et les chercheurs ne sont pas en retard... Ils le sont d'autant moins qu'ils ont amorcé ces travaux de manière concertée, organisée, novatrice depuis au moins une dizaine d'années : bel exemple d'anticipation!

\section{Références}

Abbaye Saint-Pierre de Solesmes (Ed.), 1960. Problèmes agricoles et ruraux. Présentation et tables par les moines de Solesmes, Tournai, Desclée \& cie.

Acloque Desmulier D., Gasselin P., Rouillé d'Orfeuil H. (Eds), 2014. Agricultures familiales, trajectoires, modernités et controverses (I), Revue Tiers Monde, 220, 4.

Acloque Desmulier D., Gasselin P., Rouillé d'Orfeuil H. (Eds), 2015. Agricultures familiales, trajectoires, modernités et controverses (II), Revue Tiers Monde, 221, 1.

AMIRA (Groupe de recherche pour l'amélioration des méthodes d'investigation en milieu rural africain), 1978. Enquêtes et outils statistiques. Vol 1, Le choix d'une unité, Paris, INSEE.

Aubert F., Piveteau V., Schmitt B. (Eds), 2009. Politiques agricoles et territoires, Versailles, Quæ.

Aubert M., Perrier-Cornet P., 2009. Is there a future for small farms in developed countries? Evidence from the French case. Communication à the 111th EAAE-IAAE Seminar "Small farms. Decline or persistence?", June 26-27th, University of Kent (United Kingdom).

Augé-Laribé M., 1902. Grande ou petite propriété ? Histoire des doctrines en France sur la répartition du sol et la transformation industrielle de l'agriculture. Thèse de droit, Université de Montpellier, Montpellier.

Badouin R., 1967. Agriculture et accession au développement, Paris, A. Pedone.

Badouin R., 1971. Économie rurale, Paris, Armand Colin.

Barral P., 1968. Les agrariens français. De Méline à Pisani, Paris, Armand Colin. 
Barthélémy D., 1988. La naissance de l'entreprise agricole, Paris, Economica.

Banque mondiale, 2008. Rapport sur le développement dans le monde 2008. L'agriculture au service du développement, Washington, Banque mondiale.

Benjamin C., 1996. Emploi et pluriactivité dans les exploitations agricoles. Analyse théorique et application au cas français, Paris, Economica.

Benoit-Cattin M., Faye J., 1982. L'exploitation agricole familiale en Afrique soudano-sahélienne, Paris, Agence de coopération culturelle et technique/Presses universitaires de France.

Bosc P.-M., Sourisseau J.-M., Bonnal P., Gasselin P., Valette É., Bélières J.-F. (Eds), 2015. Diversité des agricultures familiales. Exister, se transformer, devenir, Versailles, Quæ.

Boussard J.-M., 2007. L'instabilité, un phénomène accidentel ou structurel ?, in Boussard J.-M., Delorme H. (Eds), La régulation des marchés agricoles internationaux. Un enjeu décisif pour le développement, Paris, L'Harmattan.

CAS (Centre d'analyse stratégique), 2010. Les cessions d'actifs agricoles dans les pays en développement. Rapport au secrétariat d'État chargé de la prospective et du développement de l'économie numérique, Paris, La Documentation française.

Chambers R., Conway, G.R., 1991. Sustainable rural livelihoods: practical concepts for the 21st century, Institute of Development Studies Discussion Paper, 296.

Chombart de Lauwe J., 1979. L'aventure agricole de la France de 1945 à nos jours, Paris, Presses universitaires de France.

Cornu P., Mayaud J.-L. (Eds), 2008. Nouvelles questions agraires, exploitants, fonctions et territoires, Paris, la Boutique de l'histoire.

Galtier F., 2012. Gérer l'instabilité des prix alimentaires dans les pays en voie de développement. Une analyse critique des stratégies et des instruments, Paris, Agence française de développement.

Gasselin P., Choisis J.-P., Petit S., Purseigle F., Zasser S. (Eds), 2014. L'agriculture en famille: travailler, réinventer, transmettre, Les Ulis, EDP Sciences, http:/ / www.edp-open.org/ lagriculture-en-famille-travailler-reinventer-transmettre.

Gastellu J.M., 1980. Mais où sont ces unités économiques que nos amis cherchent tant en Afrique?, Cahiers OrstomSciences Humaines, 17, 1-2, 3-11.

Giraud C., Rémy J., 2008. Les choix des conjoints en agriculture, Revue d'Études en Agriculture et Environnement, $88,21-46$.

Godelier M., 1984. L'idéel et le matériel. Pensée, économies, sociétés, Paris, Fayard.

Hayami Y., Ruttan V.W., 1998. Agriculture et développement, une approche internationale, Paris, Inra.

Hervieu B., Mayer N., Muller P., Purseigle F., Rémy J. (Eds), 2010. Les mondes agricoles en politique. De la fin des paysans au retour de la question agricole, Paris, Presses de sciences Po.

Hervieu B., Purseigle F., 2013. Sociologie des mondes agricoles, Paris, Armand Colin.

Jacquet P., Pachauri R.K., Tubiana L. (Eds), 2012. Développement, alimentation, environnement. Changer l'agriculture?, Paris, Armand Colin.

Lamarche H., 1992. L'agriculture familiale, comparaison internationale. T. I, Une réalité polymorphe, Paris, L'Harmattan.
Lamarche H., 1994. L'agriculture familiale, comparaison internationale.T. II, Du mythe à la réalité, Paris, L'Harmattan.

Le Roy E., Karsenty A., Bertrand A., 1996. La sécurisation foncière en Afrique. Pour une gestion viable des ressources renouvelables, Paris, Karthala.

Loyat J., 2013. Agricultures du monde. Les questions qui font peur, les solutions pour agir durablement, Paris, France agricole.

Malassis L., 1958. Économie des exploitations agricoles. Essai sur les structures et les résultats des exploitations agricoles de grande et de petite superficie, Paris, Armand Colin.

Malassis L., 1973. Agriculture et processus de développement. Essai d'orientation pédagogique, Paris, Unesco.

Malassis L., 2001. La longue marche des paysans français, Paris, Fayard.

Mayaud J.-L., 1999. La petite exploitation rurale triomphante. France, XIX ${ }^{e}$ siècle, Paris, Belin.

Mazoyer M., Roudart L., 2005. La fracture agricole et alimentaire mondiale. Nourrir l'humanité aujourd'hui et demain, Paris, Universalis.

McIntyre B.D., Herren H.R., Wakhungu J., Watson R.T. (Eds), 2009. Agriculture at a crossroads. International assessment of agricultural knowledge, science and technology for development (IAASTD). Global report, Washington (D.C.), Island Press.

Mendras H., 1967. La fin des paysans. Innovations et changement dans l'agriculture française, Paris, SEDEIS.

Mendras H., 1984. La fin des paysans. Suivi d'une réflexion sur la fin des paysans, Le Paradou, Actes Sud.

Milhau J., Montagne R., 1964. Économie rurale, Paris, Presses universitaires de France.

Neveu A., 2012. Agriculture mondiale : un désastre annoncé, Paris, Autrement.

Nicourt C., 2013. Être agriculteur aujourd'hui. L'individualisation du travail des agriculteurs, Versailles, Quæ.

Parance B., Saint Victor J. (de) (Eds), 2014. Repenser les biens communs, Paris, CNRS.

Paul J.L., Bory A., Bellande A., Garganta E., Fabri A., 1994. Quel système de référence pour la prise en compte de la rationalité de l'agriculteur: du système de production agricole au système d'activité, Les cahiers de la recherchedéveloppement, 39, 7-19.

Pech R., 2009. Jaurès paysan, Toulouse, Privat.

Petit M., 1975. Évolution de l'agriculture et caractère familial des exploitations agricoles, Économie rurale, 106, 45-55.

Petit M., 2006. L'exploitation agricole familiale: leçons actuelles de débats anciens, Cahiers Agricultures, 15, 6, 486490.

Petit M., 2011. Pour une agriculture mondiale productive et durable, Versailles, Quæ.

Ploeg J.D. (van der), 2014. Les paysans du XXI siècle. Mouvements de repaysannisation dans l'Europe d'aujourd'hui, Paris, Charles Léopold Mayer.

Pouch T., 2010. La guerre des terres. Stratégies agricoles et mondialisation, Paris, Choiseul.

Purseigle F., 2015. De la famille à la firme : itinéraire ruraliste, in Paradeise C., Lorrain D., Demazière D. (Eds), Les sociologies françaises. Héritages et perspectives, 1960-2010, Presses universitaires de Rennes, 389-400.

Rémy J., 2010. Un métier en transformation. Introduction, in Hervieu B., Mayer N., Muller P., Purseigle F., Rémy J. (Eds), Les mondes agricoles en politique. De la fin des paysans au 
retour de la question agricole, Paris, Presses de sciences Po, 41-50.

Rouillé d'Orfeuil H., 2013. L'emploi agricole, un défi historique, Fonds documentaire dynamique sur la gouvernance des ressources naturelles de la planète, http://www.agter.org/ $\mathrm{bdf} / \mathrm{fr} /$ corpus_chemin/fiche-chemin-277.html.

Rouillé d'Orfeuil H., 2014. 2014, Année internationale de l'agriculture familiale, La Revue de l'Académie d'agriculture, 3, 32-34.

Roux B., 2014. Les transformations de l'agriculture familiale: une longue histoire qui continue à s'écrire, La Revue de l'Académie d'agriculture, 3, 35-39.

Samuelson P.A., 1967. L'économique. Techniques modernes de l'analyse économique. T. 2, Paris, Armand Colin.

Scott J.C., 1976. The moral economy of the peasant rebellion and subsistence in Southeast Asia, New-Haven/London, Yale University Press.

Segalen M., 2012. Les nouvelles formes de conjugalité : du désordre dans l'institution ?, Cahiers français, 371, 8-16.

Sen A., 1999. Development as freedom, Oxford, Oxford University Press.
Séronie J.M., Boullet P., 2007. L'exploitation agricole flexible, Les Cahiers CERFRANCE, http://blog-agri.cerfrance.fr/ blog-agri/2010/12/14/lexploitation-flexible-agricole/.

Servolin C., 1989. L'agriculture moderne, Paris, Seuil.

Small L.A., 2007. The sustainable rural livelihoods approach: a critical review, Canadian Journal of Development Studies, 28, 1, 27-38.

Sourisseau J.-M. (Ed.), 2014. Agricultures familiales et mondes à venir, Versailles, Quæ.

Sourisseau J.-M., Kahane R., Fabre P., Hubert B. (Eds), 2015. Proceedings of international encounters "Family farming $\mathcal{E}$ research", 1-3 June 2014, Montpellier, Agropolis International Edition, http://www.agropolis.org/pdf/publications/proceedings-international-encounters-familyfarming-research-june-2014.pdf.

Thirion M.-C., Bosc P.-M. (Eds), 2014. Les agricultures familiales du monde. Définitions, contributions et politiques publiques, Montpellier/Paris, Cirad/Agence française de développement, http://www.afd.fr/webdav/site/afd/shared/ PUBLICATIONS/RECHERCHE/Scientifiques/A-savoir/ 28-A-Savoir.pdf.

Reçu le 9 septembre 2015. Accepté le 7 avril 2016. 